\title{
Téoros
}

Revue de recherche en tourisme

\section{Tourisme pour tous, projet utopique ?}

\section{Marc Laplante et Roger Nadeau}

Volume 1, numéro 3, 3e trimestre 1982

Les enjeux du tourisme social

URI : https://id.erudit.org/iderudit/1080844ar

DOI : https://doi.org/10.7202/1080844ar

Aller au sommaire du numéro

Éditeur(s)

Université du Québec à Montréal

ISSN

0712-8657 (imprimé)

1923-2705 (numérique)

Découvrir la revue

Citer cet article

Laplante, M. \& Nadeau, R. (1982). Tourisme pour tous, projet utopique ? Téoros, 1(3), 2-6. https://doi.org/10.7202/1080844ar d'utilisation que vous pouvez consulter en ligne.

https://apropos.erudit.org/fr/usagers/politique-dutilisation/ 


\title{
Tourisme pour tous, projet utopique?
}

\author{
par Marc Laplante et Roger Nadeau
}

\begin{abstract}
Introduction
Le texte qui suit est consacré à une étude préliminaire sur ce qu'il est convenu d'appeler, en tourisme, les "non-partants". On y soulignera surtout la pauvreté de nos connaissances à ce sujet et conséquemment, les risques d'une actions sociale entreprise sur des bases aussi incertaines.
\end{abstract}

Auparavant, nous voulons attirer l'attention sur les concepts eux-me̊mes, c'est-à-dire sur les notions de partants et de non-partants et leurs sous-entendus. Une revue partielle de la littérature de recherche en tourisme nous a convaincus de l'existence d'une "idoIatrie" du partant. Obligatoirement, toute recherche empirique sur les comportements, les aspirations et les projets des touristes doit d'abord identifier le groupe des partants. Ce faisant, la même étude dénombre aussi les non-partants. Selon les définitions adoptées, ces derniers comptent entre $40 \%$ et $50 \%$ de la population adulte du Québec actuellement. Les promoteurs tra ditionnels du tourisme, intéressés principalement par sa dimension économique ou commerciale, tournés vers l'avenir, percoivent la situation présente comme un progrès sur la situation passée et font des extrapolations pour le futur. Le pourcentage de nonpartants est un "résidu" qu'il faudra réduire encore par des offres approprieses et un marketing spécialisé. Dans certaines provinces canadiennes $^{111}$ et dans certains pays européens $^{(2)}$, ce résidu est déjầ de $25 \%$ à $30 \%$ seulement. Selon cette même perception de la réalité, il restera toujours un sousgroupe "non-intéressant" pour les affaires, essentiellement, les pauvres.

Partir durant sont temps de vacances - ou mëme de week-end - est devenu un modèle social (un pattern), une sorte de rituel, comme jadis, la sortie du samedi soir. Conséquemment, qui ne part pas est incapable de le faire. De là à conclure que cette incapacitế était d'abord financière, il n'y avait qu'un pas qui fut vite franchi. Les premières politiques sociales du tourisme se sont construites sur cet axiome tterme défini par Robert comme "vérite indémontrable mais evidente $d^{\prime}$ un raisonnement... ". il faut aider à partir ceux qui ne partent pas.
Le tourisme social, à sa facon, sacrifie donc à la même idolâtrie. Il a fait introduire dans de grands textes internationaux bien intentionnés la notion de "droit au tourisme", en extension du droit aux congés annuels ou aux vacances, légitimant ainsi son action spécifique auprès des non-partants. En fait, comme nous le montrerons ci-dessous, le tourisme social oeuvre simultanément pour l'accessibilité de tous au loisir d'abord, aux vacances en particulier et au tourisme occasionnellement, réduisant très souvent ce dernier à une sortie prolongée de son lieu de résidence habituel à l'occasion des vacances ${ }^{13 i}$.

Nous nous proposons d'abord de questionner l'axiome: "qui ne part pas est économiquement incapable de partir". Pour nous, il s'agit d'une vérité non encore démontrée, mais démontrable par des recherches appropriées. Le programme de recherche, dont nous parlerons plus loin, est fondé sur quelques hypothèses générales dont voici les principales $^{|4|}$ :

(a) Les inégalités culturelles sont aussi lourdes que les inégalités socio-économiques pour expliquer les taux de non-départ touristique.

(b) Le tourisme est une expérience culturelle spécifique vécue actuellement par très peu de "partants".

(c) L'action d'animation et d'éducation caractéristique des agents du tourisme social ne porte pas sur l'apprentissage à l'expérience touristique proprement dite et le potentiel de développement culturel lié à de telles expériences est pré sentement très négligé.

(d) La mise en valeur de ce potentiel devient une priorité pour donner au tourisme sa raison d'être à une époque de rareté qui menace de réduire substantiellement les activités dans ce domaine toujours identífié comme un luxe.

Partir pour partir, comme des "désoeuvrés" disailt Littré dans sa définition initiale du tourisme, comme des gens fuyant pour quelques semaines une réalité quotidienne de plus en plus invivable, comme c'est le cas maintenant, ne nous apparaît pas comme une solution d'avenir. Ces départs devien- dront hors de prix, compte-tenu de leur impuissance à améliorer le sort de tous et chacun.

Les origines de notre programme de recherche remontent aux premières critiques des quelques maigres données accumulees sur les non-partants. Ces conclusions se prêtent à plusieurs interprétations. Par la suite, nous avons été sensibilisés aux résultats parfois inattendus de certaines entreprises du tourisme social. Finalement, nous avons dù soumettre au mème examen critique plusieurs conclusions de recherches sur le phenomène touristique lui-même, sur sa portée et sur ces limites.

\section{Profils des non-partants}

La catégorie des non-partants dans les enquêtes touristiques regroupe des gens de situations fort differentes. Retenons surtout les deux sous-groupes suivants: les nonvacanciers et les vacanciers non-partants. L'univers des vacances commence quand débute une période prolongée d'arrèt de travail. Un tel arrêt est dit prolongé quand il dépasse l'arrêt d'un long week-end de 3 jours. Certains individus jouissent de tels arrêts de l'activité professionnelle durant 4 ou 5 semaines chaque année, prises consécutivement ou par périodes plus courtes en diverses saisons. D'autres cependant ne prennent pas une seule pose de 4 jours consécutifs ou plus en une année. Nous nous interrogeons d'abord à propos de ces nonvacanciers.

\section{a) Douze mois sans vacances}

En 1959, 54\% des salariés du Québec n'avaient pas pris de vacances dans les 12 mois précédents l'enquête ${ }^{|5|}$. $70 \%$ de ces non-vacanciers de $1959 \mathrm{n}$ 'avaient pas pris de vacances durant les cinq années précédentes. Par contre, $75 \%$ de tous les répondants ont nié la proposition "Prendre des vacances est un luxe". II y a 23 ans au Québec, un travailleur salarié sur quatre environ connaissait des frustrations ou des privations en matière de vacances annuelles.

A cette époque, prendre des vacances était un phénomène urbain. En milieu rural, $23 \%$ des familles avaient pris des vacances en 1959, contre $73 \%$ des gens des grandes villes. Cette enquête ne nous révèle pas le pro- 
fil de ces non-vacanciers. Elle nous apprend par contre que $46 \%$ des familles sacrifiaient les voyages en vue d'épargner (c'était le premier lieu sacrifiel et même que $8 \%$ faisaient ce meme sacrifice pour boucler leur budget.

Pourquoi les familles ne prenaient-elles pas de vacances? Par ordre, nous retrouvons les raisons suivantes:

1. le manque d'argent: $36 \%$ des non-vacanciers

2. en chômage ou à la retraite: $17 \%$

3. à cause des enfants: $10 \%$

4. pas de vacances payées: $8 \%$

5. maladie: $7 \%$

6. ne peut pas quitter le travail: $7 \%$

7. n'ont pas les moyens: $6 \%$

8. autres raisons: $9 \%$

En regroupant la raison 1, avec les raisons 2,4 et 7 qui expriment toutes une incapacité financière, $67 \%$ des non-vacanciers se sont privés de vacances à cause de la faiblesse de leurs revenus.

L'enquête Montpetit-Samson de 1970 dans la région métropolitaine de Montréal révé lait que $25 \%$ des menages n'avaient pas pris de vacances cette année-là. $16 \%$ de cette population n'avaient pas pris de vacances depuis 3 ans."

La même enquête, réalisée en 1980 dans la mème région, nous apprend que le pourcentage des non-vacanciers est de $29 \%$. Cette étude est récente et présente un portrait-type du non-vacancier: $1162 \%$ des non-vacanciers de 1980 n'ont pas pris de vacances en 1979, ni en 1978; 2) $45 \%$ des non-vacanciers n'ont pas eu de congés payés en 1980; 3) parmi les salariés, $20 \%$ n'ont pas eu droit à un congé payé cette année-lă et tous se retrouvent parmi les nonvacanciers; 4) le taux de non-vacanciers ne varie pas si on compare les anglophones aux francophones, mais il atteint $34 \%$ parmi les allophones; 5) la corrélation entre le niveau de revenus et le taux de non-vacanciers est forte mais elle demande quelques commentaires. Le tableau ci-dessous regroupe les résultats de Samson et Barnier selon certaines catégories de revenus familiaux bruts annuels.
Malgré l'absence de tests de signification, nous pouvons prendre pour acquis que les non-vacanciers sont proportiennellement plus nombreux parmi les ménages de $\$ 10,000$. par année ou moins. Les vacanciers, par contre, sont nettement plus nom. breux dans la classe de $\$ 30,000$. a $\$ 50,000$. "classe qui voyage". C'est connu. Le tableau nous apprend d'autre part que $27 \%$ des vacanciers n'ont que de $\$ 10,000$ à $\$ 20,000$. de revenus et que $6 \%$ des nonvacanciers gagnent plus que $\$ 50,000$ par année. Ces quelques données suffisent à conclure que le niveau des revenus n'expli. que pas, à lui-seul, le fait de prendre ou de ne pas prendre de vacances. D'ailleurs, la même étude nous confirme cette conclusion si nous considerons le tableau ci-dessous ${ }^{(9)}$.

\begin{tabular}{ll}
\hline non-vacanciers & \\
\hline Population totale & $29 \%$ \\
\hline Age: & \\
$18-24$ ans & $38 \%>$ \\
$25-34$ ans & $32 \%$ \\
$35-44$ ans & $16 \%$ \\
$45-54$ ans & $18 \%$ \\
$55-64$ ans & $31 \%$ \\
\hline Scolarité: & \\
primaire & $44 \%>$ \\
secondaire & $28 \%$ \\
collegiale & $27 \%$ \\
universitaire & $20 \%\langle$ \\
\hline Occupation: & \\
emploi, temps plein & $79 \%\langle$ \\
emploi, temps partiel* & $30 \%$ \\
chömeur * & $56 \%>$ \\
retraité & $47 \%>$ \\
étudiant & $36 \%$ \\
entretien de maison & $36 \%$ \\
\hline
\end{tabular}

"Ces groupes occupationnels né comptant que peu de cas dans l'échantillon 163 a temps partiel, 32 chömeurs, 59 retraitús et 45 étudiantsl, il faut accepter avec beaucoup de reserves les pourcentages presentés.
Les 18-24 ans prennent nettement moins de vacances que les autres. Ils forment $15 \%$ de l'échantillon. On peut imaginer qu'ils sont aussi nettement plus nombreux parmi les $\$ 10,000$. de revenus familiaux bruts par année $^{\text {rtố. }}$. Le niveau d'études primaires, qui doit également regrouper un fort pourcentage des revenus faibles, ne favorise pas les vacances, puisqu' on y trouve des concentrations de travailleurs semi et non qualifiés." "Infin, chömeurs et retraités viennent grossir les rangs des nonvacanciers: its sont probablement aussi plus nombreux proportionnellement parmi les $\$ 10,000$. et moins de revenus.

Les non-vacanciers sont donc des jeunes, des étudiants en bonne part, des jeunes chômeurs aussi car le chômage est proportionnellement plus élevé parmi les 18-24 ans, des chomeurs et des retraités. Ces gens ont en commun des revenus plus faibles que I'ensemble de la population. Mais la question essentielle reste sans réponse à partir des données de cette étude: le fait de ne pas prendre de vacances est-il lié aux statuts particuliers ou au manque de revenus. Le tableau 8 (page 39) du rapport de Samson et Barnier nous apprend aussi que les etudiants partent autant que les autres pour des vacances à destinations multiples $116 \%$ contre $17 \%$ pour l'ensemble de l'échantillonl. Ces étudiants-touristes sont-ils plus riches que les autres? Le même tableau montre que $55 \%$ des retraités icontre $61 \%$ pour l'ensemble de l'échantillon) partent de chez eux pour leurs vacances. S'agit-il uniquement de "vieux riches"? Nous estimons que ces résultats laissent la porte ouverte à plusieurs questions concernant l'influence spécifique des revenus sur le fait de prendre ou de ne pas prendre de vacances.

Les questions 9 à 12 du questionnaire de Samson et Barnier cherchaient à connaitre les raisons de l'absence de vacances. Voici les principaux résultats ${ }^{i 2 !}$.

1. pour des raisons économiques: $44 \%$ des non vacanciers

Imanque d'argent: $30 \%$ des nonvacanciers, économie pour des choses plus prioritaires: $14 \%$ des nonvacanciers!

2. obligations familiales: $33 \%$ des non-vacanciers

3. contraintes occupationnelles: $23 \%$ des non-vacanciers

Combien ne prennent pas de vacances à cause du manque d'argent strictement, selon leur propre témoignage? $30 \%$ de $29 \%$, soit environ $9 \%$. Bref, un nonvacancier sur 10 à peu près affirme ne pas prendre de vacances par manque de res. sources financières. On sait d"autre part (page 79 du rapport) que $36 \%$ des nonvacanciers n'ont fait aucune sortie de weekend durant les 12 mois précédant le sondage et $41 \%$ n'en ont fait que quelques 
unes. Est-ce toujours â cause des revenus trop faibles?

Toutes ces difficultés d'interprétation suffisent, croyons-nous, à désirer une étude plus approfondie de la question.

b) Des vacances sur place:

En 1959, selon Tremblay et Fortin, "sur 4 familles qui prennent des vacances, 3 vont à l'extérieur et, pour la grande majorité, c'est la maniere habituelle d'agir depuis 5

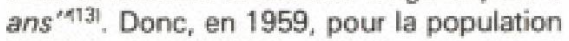
salariée du Québec, la situation est la suivante:

\begin{tabular}{ll}
\hline Non-vacanciers: & $54 \%$ \\
Vacanciers non-partants: & $11 \%$ \\
Partants: & $35 \%$ \\
\hline
\end{tabular}

Les deux études dans la région métropolitaine de Montréal auxquelles M. Samson a participe, à 10 ans d'intervalle, donnent les résultats suivants:

\begin{tabular}{lrr}
\hline & 1970 & $\mathbf{1 9 8 0}$ \\
\cline { 2 - 3 } Non-vacanciers & $25 \%$ & $29 \%$ \\
Non-partants & $15 \%$ & $10 \%$ \\
Partants & $60 \%$ & $61 \%$ \\
\cline { 2 - 3 } Total & $100 \%$ & $100 \%$ \\
\hline
\end{tabular}

On ne peut, hélas! comparer la situation de 1959 à celles de 1970 et de 1980 : les populations échantillonnées ne sont pas les mèmes et surtout, les situations dans la région très urbanisée diffèrent de celles de l'ensemble du Québec ${ }^{(14)}$.

Selon toute vraisemblance toutefois, le taux de départ en vacances s'est accru de 1959 à 1970, période durant laquelle la révolution tranquille faisait sont oeuvre. On s'étonne alors de la stabilité de la situation de 1970 à 1980: le taux de départ n'a pas varié et les non-partants sont moins nombreux aujourd'hui qu'il y a 10 ans: on reviendrait à la situation de 1959 ?

Qui sont les non-partants? Samson et Barnier les définissent comme des vacanciers qui ne s'éloignent pas à plus de 20 milles de leur lieu de résidence lles sédentaires ou "Balconville") et des vacanciers qui, tout en sortant de leur région, reviennent coucher chez-eux (l'excursionnistel. Ces derniers formaient $4 \%$ de l'échantillon en 1980 et les sédentaires, $6 \%$. Nous présentons maintenant, en résumé, les traits connus de ces deux groupes de non-partants lles fréquences sont trop faibles pour garder ces deux groupes distincts: 50 sédentaires et 36 excursionnistes).

1. Les non-partants représentent $14 \%$ des vacanciers.

2. On trouve dans ce groupe moins dianglophones $(7 \%)$ et plus d'allophones $120 \% 1$.
3. Ce genre de vacances est pratiqué davantage par les gens dans la catégorie $\$ 10,000$. aे $\$ 15,000$. de revenus familiaux bruts annuels $(33 \%)$ et nettement moins par ceux de $\$ 30,000$. et plus.

4. Les universitaires sont sous-représentés parmi les non-partants $(7 \%)$.

5. Les travailleurs semi et non spécialisés sont proportionnellement plus nombreux parmi les non-partants $(34 \%$ et $30 \%)$. On y trouve très peu de professionnels, de gérants ou d'administrateurs, de petits administrateurs à leur compte.

6. Retraités, étudiants et femmes à la maison sont sous-représentés. Les travailleurs à temps partiel sont plus nombreux. Pourquoi ces vacanciers ne partent-ils pas? $48 \%$ parce qu'ils ont d'autres occupations ou d'autres passe-temps; $32 \%$ pour des raisons économiques et $20 \%$, à cause d'obligations familiales ou de contraintes temporelles ${ }^{(15)}$,

Comme précédemment à propos des nonvacanciers, nous ne pouvons pas abonder sans réserves dans le sens des chercheurs $M$. Samson et V. Barnier quand ils affirment: "Le revenu étant un indicateur exception: nel du comportement des vacanciers, nous observons (...) que ce critere discrimine en fait les non-partants des partants. ${ }^{2161}$. Trop de facteurs interviennent en même temps sur le phénomène; sans analyse multivariée, la prépondérance du facteur revenu est difficile à établir. D'autre part, l'échantillon est trop petit dans ce cas 186 vacanciers nonpartantsl pour accorder aux pourcentages une confiance raisonnable.

\section{Des recherches pour l'action}

Pour les non-vacanciers et les non-partants, mais aussi pour les partants, nous ne pou. vons, la plupart du temps, que décrire des situations sans vraiment les expliquer. Nous observons des comportements; il reste à les comprendre. Les agents du milieu, pressés de réaliser des choses visibles et réelles, sont tentếs de se satisfaire de ces monographies sommaires et des quelques essais d'explication des réalités. Le sens commun, si utile en maintes circonstances, peut aussi devenir un piege empechant les regards neufs sur des phénomènes qui semblent évidents. Mais un comportement est toujours orienté et social; il exprime des réponses à des désirs et à des contraintes; il est guidé par des normes, des valeurs, des idéologies. Le fait brut (real fact) n'existe pas. Les motivations sont à prendre en compte constam. ment, ce qui n"est jamais chose facile et rapide. Que les gens d'action ne s'impatientent pas trop: en recherche aussi, nous aimerions que les choses aillent mieux et plus vite, que les "détails" ne nous retardent pas. Pourtant, dans un domaine relativement neuf comme le tourisme, les détails comptent.
Aussi, en aoūt dernier, des étudiants du département de géographie de l'Université de Sherbrooke ont effectué une étude de motivation auprès des Québécois sêjournant sur les plages de Wells, dans le Maine. Un répondant sur sept a justifié sa présence à Wells parce qu'il pouvait y amener son chien... Faut-il dire que les choix de répon. ses ne prêvoyaient pas celle-lă! Détail, s'il en est un, mais significatif. En première analyse, nous pouvons accumuler un grand nombre d'éléments d'explication de ce genre. Par la suite, cependant, il faut savoir interpréter tout cela. L'exemple du chien mériterait qu'on $s^{\prime} y$ arrête un peu. Une entrevue en profondeur avec ces vacanciers québécois du Maine nous apprendrait peutêtre quelle place tiennent les animaux domestiques dans la vie de tous les jours à notre époque; de la, il faudrait associer cela soit à un style de vie, soit à un contexte social (solitude, par exemple ou baisse de la vie familiale) etc. Penser à son chien au moment de son choix d'un genre et d'un lieu de vacances, c'est comme penser à ses enfants, à ses parents ägès, etc. Durant ces temps dits libres, chacun cherche la voie du compromis entre ses intérêts et ses obligations.

L'accessibilite aux vacances et au tourisme doit donc se comprendre comme toute tentative pour faire que la réalisation d'un projet de vacances ne soit pas aussi ardue qu'une course à obstacles... Ces obstacles seront toujours trop nombreux et trop difficiles pour tous, y compris pour ceux et celles qui ne semblent pas avoir de misères financières. La revue Psychology Today s'est livrée à une enquête très psychologique en 1980 sur les attentes, les satisfactions, les frustrations et les rêves de vacances: ${ }^{[171}$ On $y$ apprend des choses étonnantes à propos des gens qui ont fait apparemment de très beaux voyages. Par exemple, $11 \%$ des touristes se sentent et se disent dépressifs après leurs vacances! $27 \%$ des répondants prendraient de un a six mois de congé à leur frais, s'ils le pouvaient, pour leur développement personnel, "pour developper de nouvelles habilités (skills), des nouvelles aptitudes intellectuelles lintellectual abilities), etc. " $\mathrm{A}$ defaut de tels longs congés ll'année sabbatique est réservée à quelques heureux privilégiési, les gens comptent sur leurs vacances annuelles pour se donner toutes ces capacités désirées. Et pour se reposer aussi. Donc il ne faut pas s'étonner si les vacances et les voyages actuels laissent insatisfaits, mème les par. tants avec de bons revenus. Une action d'animation en tourisme pourrait se concevoir pour à peu près tous les palliers finalement.

Voici un témoignage d'une des lectrices de Psychology Today: "Je n'ar jamais eu de vraies vacances, je ne sais pas encore ce que c'est et pourtant, j'ai essayé... Mais les tracas de la famille, des finances, les déci. sions a prendre constamment au cours du 
voyage, tout cela fait que je ne me suis jamais senti "Wibere" (vacated) de la vie quotidienne harassante. J'ai simplement amené mes problèmes avec mor" cha

De l'importance des animaux domestiques au stress de la vie quotidienne, le spectre des besoins qu'on tente de satisfaire à l'oc casion des vacances est étonamment vaste. Que faut-il offrir à tous ces gens pour leur permettre de satisfaire le mieux possible leurs besoins? Des sous certes. Mais ceci ne résoudra pas tous les problèmes, loin de là. II faut bien d'autres choses.

L'étude de Michel Charette en 1974 faisait intervenir une nouvelle facette: "Nous déstrons mettre en évidence un autre facteur essentiel a laccessibihite aux vacances: C'est le type de perception qu'on en a. II est évident que pour une famile n'ayant jamais connu /exaltation promise par des vacances a la campagne, en vovages, la possibilité, l'éventualté de vacances niont pas la mème répercussion chez-elle que chez une famile pour laquelle l'eté au cha: let est perspective aussi naturelle que d'avor à gagner sa vie. Les habitudes culturelles face aux vacances peuvent varier considerablement et influencer par le fait mème les décisions à cet égard. "A191 Cette observation rejoint celles que signalait, lors du congrès du BITS de 1980 , le récréolo. gue Claude Cousineau de I'Université d'Ottawa qui affirmait que la non-participation aux vacances pouvait sans doute s'expliquer autant par des critères culturels et psychologiques que par des critères strictement economiques. "ll y a des personnes qui sont "éduquées" pour les loisirs et d"autres qui le sont moins. Et parmi les gens a faible revenu, on peut affirmer que l'ensemble n'est pas émotionnellement éduque au loisir. On est "loisirement" pauvre; on vit dans un univers restreint et il est évident que ce n'est pas un revenu phus elevé qui pourra combler cette lacune. Etre éduqué au loisir signitie d'abord une prise de cons. cience de limpact du loisir sur soi. C'est aussi être capable de faire des choix judi. cieux et rentables en terme de satisfaction et de croissance personnelle. C'est savoir sinformer sur les opportunites de loisirs et c'est surtout /acquisition d'une certaine confiance en soi qui permettra à lindividu de risquer et d'explorer constamment de nouvelles experiences de loisir... "201 Que d'intéressantes perspectives à explorer plus avant! ... car on pourrait peut-être arriver ainsi à la conclusion que les non-partants ont peut-être plus besoin de la part des orga nismes de tourisme social d'un ensemble de services, d'animation, d'orientation, d'information, d'aide pour la prise en charge de soi, pour l'organisation de leurs vacances plutốt qu' un simple support financier.

Bien sùr, il est possible que le praticien oeuvrant au sein des milieux défavorisés et étant en contact presque quotidien avec ces populations reste plutôt froid devant ce questionnement, estimant que les besoins sont tellement nombreux et urgents quil faut arrêter de parler et de cogiter pour passer aux actes car comme le concluait l'étude Samson-Barnier, "les comportements touristiques de la population de Montréal n'ont pas bouge beaucoup depuis les 10 demieres années et /'inaccessibilité aux departs en vacances demeure une realite pour environ $40 \%$ des gens". 21 .
Passer aux actes, c'est bien, sauf qu'il faut poser les bons gestes, prendre les décisions les plus rentables, autant du point de vue social qu'économique. Ainsi, si on regarde la clientéle qui se prévaut effectivement des services offerts par des organismes carrément orientés vers le tourisme social et partiellement subventionnés par les deniers publics, tel que la Sociéte VacancesFamilles, ou les Agricotours, ou certaines bases de plein air, on constatera que les clas. ses sociales les plus démunies économiquement sont assez peu représentées, ce qui pourrait signifier que les produits offerts répondent peu aux besoins réels et qu'ils contribuent très peu à faire partir en vacances ceux qui ne partaient pas avant. On peut poser le problème autrement: pourquoi les classes défavorisées ne sont-elles pas ou peu intéressées à prendre des vacances à la ferme malgré le fait que les coûts en soient relativement peu élevés? La réponse est-elle économique ou culturelle, ou de besoins ressentis, ou de valeur des individus, ou de perception....?

Malgré toutes ces questions qui restent en suspens, on sent le besoin de pousser plus avant les programmes, de les développer et de les multiplier afin que l'accessibilité aux vacances pour tous devienne une réalité. Encore récemment, suite à sa nomination, le nouveau ministre du Loisir de la Chasse et de la Pèche, M. Guy Chevrette, annoncait que sa prionité était de donner suite au projet d'implantation de Villages-VacancesFamilles....

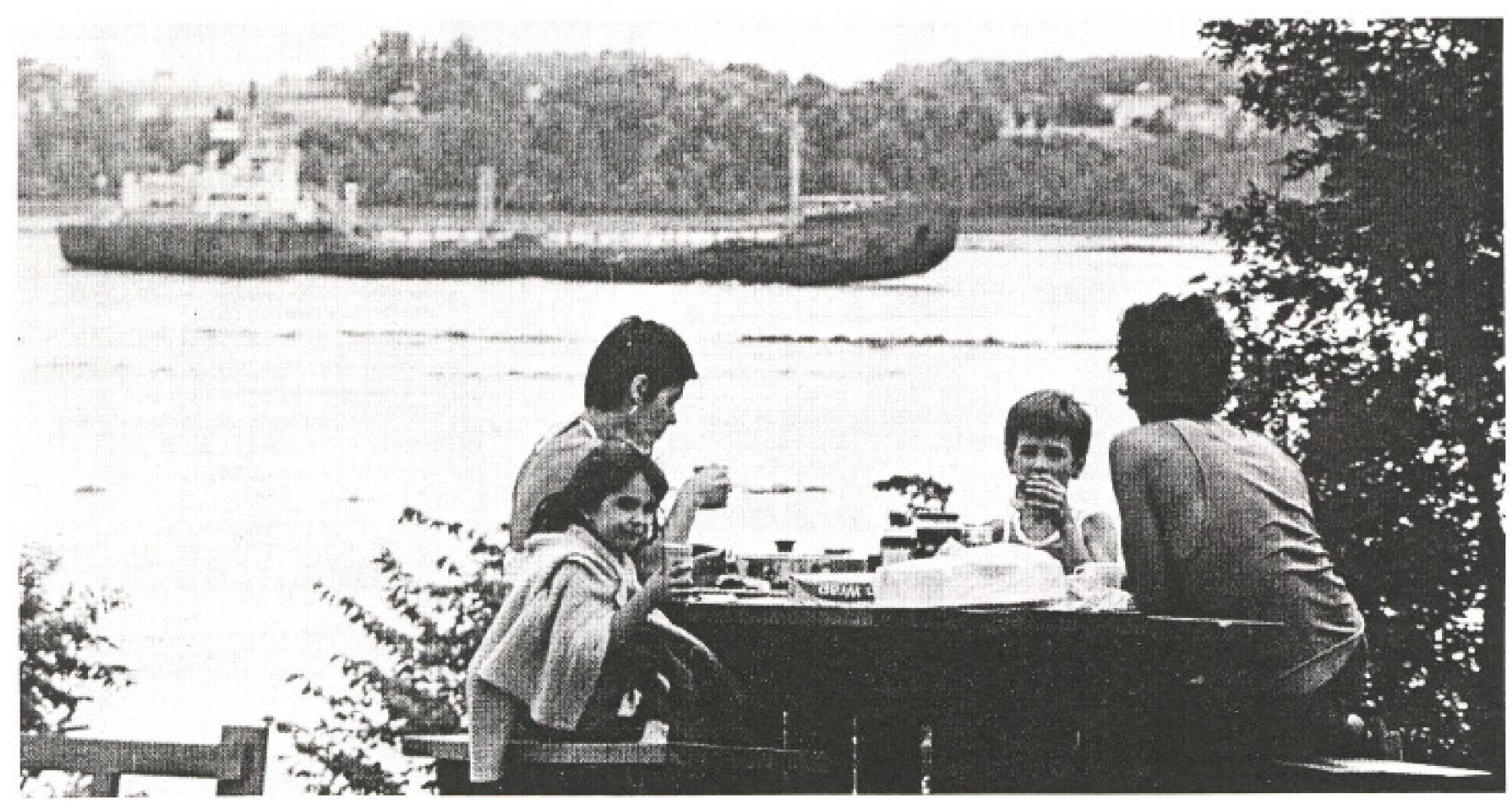




\section{Conclusion}

Les co-signataires de cet article ont conscience de la tăche qui les attend au cours des trois prochaines annees. Ils savent combien il est plus facile de pointer ce qui ne va pas que de proposer des solutions neuves. Après avoir parcouru une abondante littérature depuis quelques annees, c'est delibérément qu'ils ont choisi d'étudier en priorité les clientèles-cibles du tourisme social. D'autres grands thèmes méritaient aussi d'être ré-examinés par la recherche. Mais, d'un point de vue socio-politique, nous sommes persuadés que les années de grăce achèvent pour le tourisme social. Bientôt la période d'essais et erreurs sera passée et on lui demandera de faire la preuve de la pertinence de son option. Ses défenseurs doivent dès maintenant s'engager dans un vaste effort de prospective à la Gaston Berger. Voir loin, voir grand, penser humain, prendre des risques. ${ }^{1221}$

Nous nous associerons a cet effort par un programme de recherche sur les caractéristiques et les besoins des diverses clientèles du tourisme social et spécialement imais non exclusivement/ des non-partants et des non-vacanciers.

Dès cette année, en octobre et novembre 1982, un premier sondage téléphonique auprès de 3,000 ménages québécois reprendra la plupart des thèmes abordés par l'étude de Samson-Barnier. Le questionnaire sera administré à un échantillon de la population de chacune des 18 régions touristiques du Québec. Des éléments nouveaux seront également introduits dans le questionnaire: une mesure subjective de l'expérience touristique vécue pour les partants, une grille plus complète des conditions de vacances tavoir ou non des conges annuels payés, pouvoir prendre ou non des vacances à ses frais, pouvoir ou non choisir ses dates de vacances, aménager son temps de congé, etcl, quelquess questions sur les niveaux de satisfaction, quelques autres sur le processus de prise de décision quant au choix d'un genre de vacances, etc.

Ce sondage est une exploration. Parallèlement, nous réaliserons des entrevues libres en profondeur avec quelques cas-types de chaque catégorie de population Inonvacanciers, non-partants, partants, etc). II s'agit de concevoir et de mettre à l'épreuve les outils qui serviront aux entrevues de la phase 2, en 1983-84. Mille personnes, choisies parmi les répondants de la première année, seront visitées en 1983. Les questionnaires comprendront des questions ouvertes et les données qualitatives seront traitées ensuite par les techniques de l'analyse de contenu. Le cadre théorique, en voie d'élaboration, tente de concilier plusieurs thèses en vogue actuellement comme celle de l'acculturation ${ }^{1231}$ et celle du tourisme comme facon, pour le profane, d'explorer et de comprendre les mécanismes de la société moderne et le caractère hétérogène de notre monde complexe ${ }^{\mid 241}$.

La troisième phase du programmme de recherche, de l'automne 1984 au printemps 1985, prévoit une consultation systématique avec les intervenants en tourisme du Québec et plus spécialement avec ceux du tourisme social. II s'agira d'une première mise à l'épreuve des résultats des deux années précédentes de recherches. L'objectif de cette troisième étape est d'étudier les rapports entre les services offerts et les besoinsattentes des diverses clientèles touristiques du Québec. Les recommandations décou- leront des résultats de cette consultation réalisée selon les exigences de la rechercheaction.

Soulignons en terminant que le programme de recherche prévoit aussi la meilleure diffusion possible des résultats de nos travaux à chacune des étapes. Le numéro de juin dernier de Téoros soulignait l'urgence de la recherche en tourisme; les prochains numéros apporteront aux lecteurs nos premiers résultats.

\section{Notes}

(11) Olfice du Touisme du Canada Les voyages des canadiens en 1977 Traveldata Limited. En 1977, le taus de départ par province trait: Atlantique: $43 \%$, Gubtex $51 \%$, Ontario $59 \%$ Praires: $59 \%$, Colombie Briannique: $70 \%$. p. 11

(2) En $1977,72 \%$ des menages amescains ont bit un woyege de varances de $160 \mathrm{lmm}$ et plus. En 1909, probs de 70 \% des thes dents de Scandinswe acrient fist un rowage a loccason de vacances. OM.T. Teurisme Mondial . World Travel, 1979

9) "Il est appatu que le tourisme tasit l'une des composantes du loist et que if theme du "bourime social pous tous" allat arababiement deverir celui des "aisirs pour tous". R. LAN

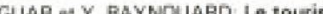
me social. Que sais.

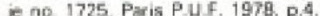

(4) Progranme de tormation de chercheus et do action concer tev If.C.A.C.I. numbe EO-2065. Miristatre de l'éducation du Oufbec pept de trois ans intite Tourisme social: caras téristiques et besoins des diverses clientales, incluant spécialement les non partants. Les co-drecteurs co ce projet san Roger Nadeau et Marc Laplante. Cinq fitudiants do 2ieme crcle i3 de Sherbbrooke et 2 de rUQAMI composen l'équipe de recherche. Plusieurs associations touristiques répionales (A. T.R.I ant accapte de participer a la cueillette des donnders.

(5) TREMELAY, M.A., FORTIN, G evee la callathontion de M. LAPLANTE. Les comportements teonomiques de le famille salaride du Québec, Quebec. Presce de runme: sine Laval, 1964, a. 209.

NE. Las sulaiss a ce moment la representaient 61 \% des

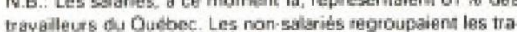

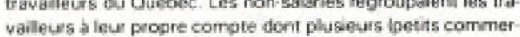

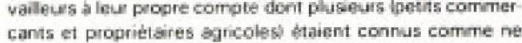
prensant pas de yacances. Le pourcentage de non-vacanciers

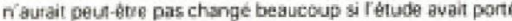
sua lansambere de la main-d cenure.
46) Idem p. 211. A note: ces categories de reponses ont eté élabotetes apres les entrevues, par les codficateurs, à partir or une snalyse de consern des réponses a une question ouverte.

17) MONTPETIT, Marc et SAMSON, Mancel: Vacances at tou. risme. Montréal, Los Pressens de liUniversite du Quebec, Les Cahiers of C.A.U.R. No. 3. 1972. p. 30

18 SAMSON, Marcel et BARNIER, Veronique, Vacances at tourisme 1980. Mentreal, I.N.R.S. Urbanisation, detudes el documents Na. 30, 1982 p. 39

(9) Idem, tableaur, psge 36 et page 39

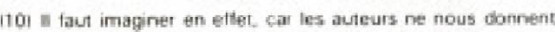
gas tous les tobisauk statistiques de base pour observer ces correlations.

(11) SAMSON, M. et BARNIER, V., op cit. p. 29 Tableau 3.

(12) Voir note 10 ci-haut in nous taut estimer des chiftres à partir

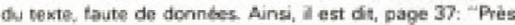
des deux tiers de ceux qui ont apporé des raisens deonami-

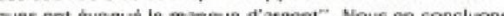
que les deus tiers do $44 \%$ dennent environ $30 \%$ ?

QNe les deus liers de 44 wo donnent environ 30 .

114) Par ensmple. Ienquete du Hast Commissariat a la jeunesse aux loisirs et sux sports du Quebec de 1978: Participation des quebeccois aux activiths de loisir nous apprend gup ce sont les gens des ribgions les plus técignees qui font davan-

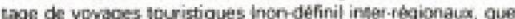

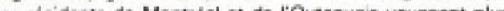

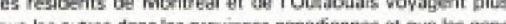
que les auros dans hespomincescanacien gens des Cantons de l'Est et de Montrisl sontem plus"que les autres hors du Cenads Les habitudes touristiques sembiem varier diune region a li sutre et surtout, seion qu'an habive $\rightarrow$ Montribal ou silleus. Tabledu 27, a. 86 (15i SAMSON, M et BARNIER, V., op. cit. p. 40 b 44 et tabieguir 8 a 12 inelusivement.

116. Idem, 0. 40. LAURENT, Alain, dans Libérex las vacances [Seuil. 19731, rapocrte, page, 27 une enquete résumé dans le Monde du 5 juiliet 1972 selon laquelle $70 \%$ des individus ne partant pas en vacances ne le regretient pas trop.

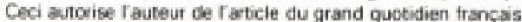
I.P. Cuelinl. deremette en cause la notion de "partant"

117 CAFIN, Autbenstein "Psychologr Todar's Surver Aeport on How Ameicans Vow Vacations". Psycholony Today, mai 1980. Zit Davis Publishing Co

116\% Idem. p. 121

119. CHARETTE, Miehel: Aappon Swnthèse II, 1974: Campsgne des téderations du grand Montetal, p. 69

(20) BITS: Ragpan du Congès 1980, Quebec, p. $413-414$

Q11 SAMSON, $M$ EI BARNIEA, $v$, op. eit. p. 21

(22) BEAGEA, Gasion, Phemamenalogie du temps ot perspect ves. Paris, P.U.F, 1964

(23) BoYER, Marc Le tourisme, Paris, Seuil, 1972; SNITH, V leds: Host and guests: the anthropology of tourism. 1976, Graburn N. H.H. ledt. Ethnic and tourist arts: Cultural Expressions from the Fourth World, Derkeley, Universily of Callemial Press, 1976

(24) Cest essernielement la these stimulante et controwerste de MACCANNEL Dean. The Tourist: a new Theory at the Leisure Class. New York, Shocken looks, 1976. 